\title{
Lineamientos del plan de acción de la SER en el bienio 2020-2021
}

\section{Features of Chilean Respiratory Society actions for the 2020-2021 biennium}

Primeramente quiero agradecer al Dr. Hernán Cabello y al directorio saliente todo el apoyo que me han otorgado, como también las enseñanzas que me han brindado, durante los dos últimos años en que desempeñé la vicepresidencia de la Sociedad Chilena de Enfermedades Respiratorias- SER. Además, debo felicitar y agradecer al nuevo directorio que me acompañará durante los años 2020 y 2021, periodo en que me desempeñaré en la presidencia de esta sociedad. En este bienio enfrentaremos el gran desafio de continuar con el excelente trabajo del directorio anterior, en un ambiente nacional impactado y conmocionado por la crisis social iniciada el 18 de octubre del año pasado y que aún está en desarrollo. Trabajaremos mancomunadamente en equipo en función de lograr el propósito fundamental de la SER -establecido en sus estatutos- que es contribuir al desarrollo en Chile de las disciplinas de las ciencias médicas y de las ciencias de la salud para mejorar la salud respiratoria de nuestro país.

Las principales estrategias que propongo para cumplir con los objetivos de la SER son las siguientes:

1. Promover y respaldar la investigación en el campo clínico, básico y epidemiológico, incluyendo la necesaria capacitación principalmente de los socios más jóvenes, apoyando los proyectos y postulaciones a los concursos de investigación, el fortalecimiento de redes de investigación y la difusión de los resultados en jornadas cientificas y publicaciones.

2. Contribuir a la formación continua de los profesionales de la salud en las diversas áreas del conocimiento de las enfermedades respiratorias a través de las jornadas y congresos científicos, los cursos de postítulo, la revista de la Sociedad, talleres y seminarios de temas especificos.

3. Respaldar activamente el trabajo regional de las filiales de la SER, como también el trabajo activo de todas las comisiones, porque tanto las filiales como las comisiones son fundamentales para el quehacer cientifico y de extensión de la SER tanto a nivel regional como nacional.

4. Contribuir a que la Revista Chilena de Enfermedades Respiratorias, órgano oficial de difusión cientifica de nuestra sociedad, siga avanzando en su proceso de modernizar y profesionalizar su gestión como también fortaleciéndose cada vez más, a través de incentivar la publicación de artículos de calidad provenientes tanto de Chile como del extranjero, con la colaboración activa de los miembros de la SER en la generación y revisión de publicaciones de investigaciones, de casos clínicos, de actualizaciones temáticas y de guías o normas de manejo de las enfermedades respiratorias, apoyados por todas y cada una de las comisiones de la SER. Al respecto, debo hacer presente que entre los deberes que contrae un profesional al incorporarse a la Sociedad, está la obligación de actuar oportuna y eficientemente como revisor de manuscritos, cuando el Comité editorial de nuestra revista se lo solicite.

5. Incorporar a la SER a más socios médicos tanto especialistas como no especialistas, como también los profesionales de la salud no médicos como kinesiólogos, enfermeros, tecnólogos-médicos y otros afines a nuestro quehacer en enfermedades respiratorias y que esperamos que inmediatamente después de su ingreso a la SER, se incorporen activamente al trabajo de las comisiones y filiales favoreciendo el trabajo colaborativo interdisciplinario. 
6. Fortalecer y modernizar las redes de comunicación de la SER, incorporando cada vez más las plataformas digitales que facilitan la comunicación para las reuniones, tanto de trabajo como de formación continua.

7. Mantener y fortalecer la comunicación y el trabajo conjunto con sociedades cientificas de Chile y de otros países, principalmente dentro de América Latina, como también con las sociedades dedicadas a enfermedades respiratorias en EE. UU. y de Europa y otros continentes, permitiendo que podamos trabajar coordinadamente en el campo de la investigación, extensión y promoción de la salud.

Creo que a los lineamientos generales que he planteado en esta propuesta, se sumarán las ideas que ciertamente surgirán del trabajo en equipo de los socios, generando las mejores estrategias que permitan que la SER sea un gran aporte para la salud respiratoria en nuestro país y que continúe siendo reconocida como una entidad cientifica de referencia dentro y fuera de Chile.

Dra. Laura Mendoza Inzunza

Presidenta, Sociedad Chilena de Enfermedades Respiratorias Email: presidencia@serchile.cl 